\title{
OMAE2008-57254
}

\section{APPLICATION OF A VOF METHOD TO MODEL COMPRESSIBLE TWO-PHASE FLOW IN SLOSHING TANKS}

\author{
Rik Wemmenhove \\ Department of Mathematics \\ University of Groningen \\ PO Box 407, 9700 AK Groningen \\ The Netherlands \\ Email: r.wemmenhove@math.rug.nl \\ Arthur E.P. Veldman \\ Department of Mathematics \\ University of Groningen \\ PO Box 407, 9700 AK Groningen \\ The Netherlands
}

\author{
Roel Luppes \\ Department of Mathematics \\ University of Groningen \\ PO Box 407, 9700 AK Groningen \\ The Netherlands
}

\begin{abstract}
The growing transport of $L N G$ in partially filled tanks raises the demand to have accurate methods to predict the fluid behaviour in these sloshing tanks and the effect of the sloshing fluid on the tanker motion. To examine the motion of the sloshing fluid, model experiments have been carried out on a scale of 1:10. Different tank filling ratios and types of motion have been tested to study the sloshing fluid behaviour for various sea states.
\end{abstract}

The model experiments have been carried out to provide extensive validation material for the numerical method COMFLOW. The details of this improved Volume Of Fluid (iVOF) method are presented in the paper. The method resolves the governing equations in both fluids, one of them being compressible. The compressibility of the second phase is especially important for more violent flow conditions, when two-phase phenomena such as air entrapment and air entrainment occur frequently. Particular attention in the numerical method has been paid to the treatment of the flow variables around the interface, especially the density. The fluid is convected by means of a first- or second-order upwind scheme.

The behaviour of the sloshing fluid strongly depends upon the regularity of the tank motion and the filling ratio of the tank. Video frames, wave probes and pressure transducers have been used to compare the fluid flow of simulation and experiment. Two-phase effects such as air entrapment are more common for increasing tank filling ratios and for more irregular tank motion. A realistic simulation of these effects is possible by modeling two-phase flow, especially when using a relatively fine grid and applying the less-dissipative second-order upwind scheme.

Compared to the earlier paper on the numerical simulation of sloshing in LNG tanks [8], where the numerical method was validated for regular sway motion, more extensive attention is paid to the accuracy of the applied discretisation schemes in space and time. The results of different schemes are now evaluated for both regular and irregular sway and roll motion of LNG tanks. 


\section{NOMENCLATURE}

$\begin{array}{lll}\mathrm{dt} & {[\mathrm{s}]} & \text { time step } \\ \mathrm{F} & {[\mathrm{N}]} & \text { body force } \\ F_{b} & {[-]} & \text { open cell fraction } \\ F_{s} & {[-]} & \text { liquid cell fraction } \\ \mathrm{g} & {\left[\mathrm{ms}^{-2}\right]} & \text { gravity vector } \\ \mathrm{h} & {[\mathrm{m}]} & \text { grid cell spacing } \\ \mathrm{n} & {[-]} & \text { time level } \\ \mathrm{p} & {[\mathrm{Pa}]} & \text { pressure } \\ \mathrm{u} & {\left[\mathrm{ms}^{-1}\right]} & \text { velocity } \\ \mathrm{V} & {\left[\mathrm{m}^{3}\right]} & \text { volume } \\ \gamma & {[-]} & \text { polytopic coefficient } \\ \mu & {\left[\mathrm{kgm}^{-1} \mathrm{~s}^{-1}\right]} & \text { dynamic viscosity } \\ \rho & {\left[\mathrm{kgm}^{-3}\right]} & \text { density }\end{array}$

\section{INTRODUCTION}

During all types of weather conditions, partially filled LNG tanks are facing varying pressure loads on their walls. Especially during more violent weather conditions, the interaction between ship movement and the movement of LNG inside tanks is worthwile to investigate. The fluid distribution in the tanks strongly depends on both the tank filling ratio and the weather conditions the tanker is subjected to. Inside the tanks, the fluid is generally a complex mixture of different fluids, with a strong mixing for violent flow conditions.

The simulation of the fluid behaviour in LNG tanks shows an analogy with the simulation of hydrodynamic wave loading on other offshore structures, so similar models can be used. Most models focus on specific aspects of free-surface flows, such as wave impact of aerated flows on walls or the velocity field under breaking waves.

As soon as flow conditions are getting more violent, using a two-phase flow model is strongly recommendable. However, the small spatial and temporal scales of entrapped and entrained air in the flow are a serious problem (see figure 1).

Existing two-phase models mainly focus mainly on single bubbles or on quite regular waves. Scardovelli and Zaleski [5] give a nice overview of existing two-phase flow models. During the last few years, there is much progress in the simulation of two-phase flows [1], [4], [9]. Furthermore, computer power is getting cheaper, increasing the possibilities to simulate large problems. Keeping track of the dynamics of the air phase in sloshing experiments is difficult. The required resolution in combination with the long time series induces the need to use powerful computers, but even then long calculation times remain an issue.

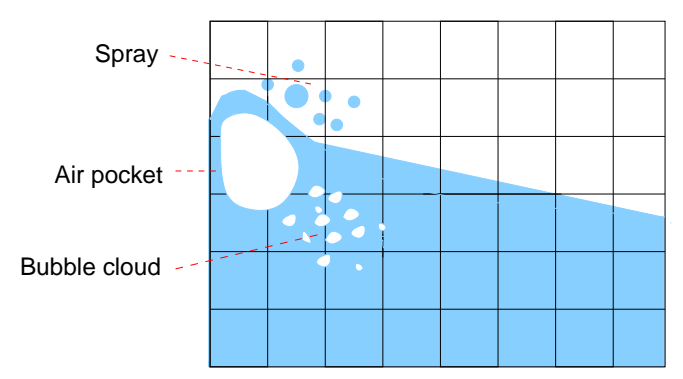

Figure 1. Two-phase phenomena at a breaking wave. Air pockets are difficult to 'catch' within the grid, bubbles and spray are almost impossible to track as individual entities.

The present paper shows the results of two-phase numerical simulations of sloshing in a tank. The simulations are validated on a series of 1:10 scale sloshing model experiments. In comparison with earlier sloshing model experiments [7], the current validation experiments are on a larger model scale. Moreover, the numerical model is able to use the experimental global motion time-traces from the sloshing tank as input for the simulations.

In the present paper the two-phase numerical model is presented. The model is aimed to simulate a wide range of offshore problems, but in this paper only the sloshing application is described.

The first section of this paper shows the governing equations the model is based on. After that, the main aspects of the discretisation of the numerical model are shown. Special attention is paid to the compressibility of the compressible phase and to the proper calculation of the density. In the last section the results of the validation of the numerical model on the sloshing experiments are shown.

\section{GOVERNING EQUATIONS}

For each point in the domain the fluid motion is governed by the continuity equation and the momentum equation. Both equations are aggregated, i.e. one set of equations is used to describe the behaviour of the two different fluids together.

$$
\begin{array}{r}
\frac{\partial \rho}{\partial t}+\nabla \cdot(\rho \mathbf{u})=0 \\
\frac{\partial(\rho \mathbf{u})}{\partial t}+\underbrace{\nabla \cdot(\rho \mathbf{u u})}_{\text {convection }}+\nabla p-\underbrace{\nabla \cdot(\mu \nabla \mathbf{u})}_{\text {diffusion }}-\rho \mathbf{F}=0
\end{array}
$$

with an external body force $\mathbf{F}$, velocity $\mathbf{u}$, pressure $p$, density $\rho$ and dynamical viscosity $\mu$.

For incompressible flow conditions the velocity field is 
divergence-free, reducing the continuity equation to

$$
\nabla \cdot \mathbf{u}=0
$$

The continuity equation and momentum equation are, after semidiscretisation in time, combined to the pressure Poisson equation to compute the new pressure value and velocity field. This pressure equation is shown in the next section.

\section{NUMERICAL MODEL}

The numerical model has been implemented in a $3 \mathrm{D}$ VOF Navier-Stokes solver called COMFLOW. Compared with the original VOF algorithm [3], a local height function improves the treatment of the free surface. The program has been developed initially by the University of Groningen to study the sloshing of liquid fuel in spacecraft [2], [6]. The micro-gravity environment requires a very accurate and robust description of the free surface. In close cooperation with MARIN (Maritime Research Institute Netherlands), this methodology was later extended to the calculation of green water loading on a fixed bow deck. Later on, the range of applications has been extended to impact loads on fixed structures [4], anti-roll tanks and sloshing tanks [8].

\section{Discretisation of the Navier-Stokes equations}

The discretisation of the Navier-Stokes equations is done on a staggered Cartesian grid, which means that the pressure is set in the cell centers and the velocity components in the middle of the cell faces between two grid cells, see figure 2. For compressible

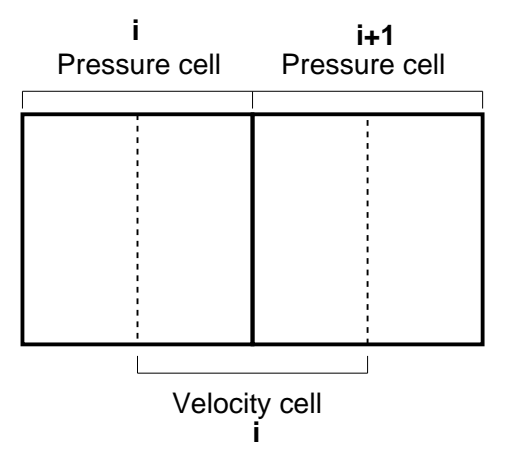

Figure 2. Staggered Cartesian grid; pressures and densities in cell centers and velocities at cell faces. The continuity equation is calculated in pressure cells, the momentum equation in velocity cells.

flow conditions, the Navier-Stokes equations, as given by eq. (1) and eq. (2), are discretised in time in principle by means of the first order Forward Euler method:

$$
\begin{gathered}
\frac{\rho^{n+1}-\rho^{n}}{d t}+\rho^{n} \nabla \cdot \mathbf{u}^{n+1}+\mathbf{u}^{n} \nabla \rho^{n}=0 \\
\frac{\mathbf{u}^{n+1}-\mathbf{u}^{n}}{d t}+\frac{\mathbf{u}^{n}}{\rho^{n}} \frac{\rho^{n+1}-\rho^{n}}{d t}+\frac{1}{\rho^{n}} \nabla \cdot\left(\rho^{n} \mathbf{u}^{n} \mathbf{u}^{n}\right) \\
+\frac{1}{\rho^{n}} \nabla p^{n+1}-\frac{1}{\rho^{n}} \nabla\left(\mu^{n} \nabla \cdot \mathbf{u}^{n}\right)-\mathbf{F}^{n}=0
\end{gathered}
$$

with $n$ the old time level and $n+1$ the new time level. The discretisation of the convective term $\frac{1}{\rho^{n}} \nabla \cdot\left(\rho^{n} \mathbf{u}^{n} \mathbf{u}^{n}\right)$ in eq. (5) requires specific attention, as this term dominates for momentumdriven applications. Figure 3 shows several spatial schemes that can be used to discretise the convective term. Applying

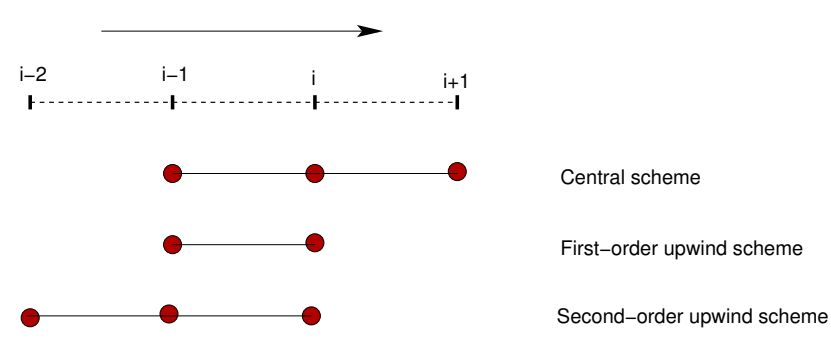

Figure 3. Schemes for spatial discretisation. $\frac{d \phi_{i}}{d x}=f\left(\phi_{i-1}, \phi_{i}\right)$ for first-order upwind and $\frac{d \phi_{i}}{d x}=f\left(\phi_{i-2}, \phi_{i-1}, \phi_{i}\right)$ for second-order upwind.

the central scheme, information is used from one upstream and one downstream velocity point. For the first-order upwind (B2) scheme, only one upstream point is taken into account, while the second-order upwind (B3) scheme uses information from two upstream points.

The B2 scheme requires a large amount of artificial diffusion to obtain a stable solution, this artificial diffusion may lead to an undesirable amount of damping of the fluid motion. Using the second-order upwind (B3) scheme, the required amount of artificial diffusion is smaller, resulting in less fluid motion damping. However, an alternative time discretisation, that will be described below, is needed to generate stable solutions when using the B3 scheme.

\section{Time integration method}

To discretise the governing equations in time, two time integration methods have been considered. The first-order Forward Euler method, as used thus far, only uses information from the previous time level $n$ to calculate a variable $\phi$ on time level $n+1$, 


$$
\phi^{n+1}=\phi^{n}+\Delta t f\left(\phi^{n}\right)
$$

An alternative for the Forward Euler Method is the second-order Adams-Bashforth time integration method. For this method, the older time level $n-1$ is considered as well in the time integration:

$$
\phi^{n+1}=\phi^{n}+\Delta t f\left(\frac{3}{2} \phi^{n}-\frac{1}{2} \phi^{n-1}\right)
$$

Both time integration methods are limited in their stability by two quantities: the CFL number $\eta=\frac{u \Delta t}{\Delta x}$ and the diffusive number $d=\frac{2 k \Delta t}{(\Delta x)^{2}}$.

The stability limits for the described time integration methods are worthwhile to investigate. Aside from the time integration, the stability limit also depends on the spatial discretisation. For most offshore test cases convection dominates the solution and in practice the CFL number $\eta$ controls the stability limit. Also, the diffusive parameter $d$ has to be smaller than one to have a stable solution for zero (or low) flow velocities, but this is mostly easily satisfied. In table 1, the limits on the CFL number are summarized for both time integration methods in combination with two upwind schemes. As visible in table 1, the Forward Euler

\begin{tabular}{||l|l|l||}
\hline \hline CFL number limit $\eta_{\max }$ & B2 upwind & B3 upwind \\
\hline Forward Euler & $1-d \approx 1$ & $\sqrt{d} \ll 1$ \\
\hline Adams Bashforth & $\frac{1}{2}-d \approx \frac{1}{2}$ & $\frac{1}{4}-\frac{1}{2} d \approx \frac{1}{4}$ \\
\hline \hline
\end{tabular}

Table 1. Limit on CFL number for different spatial upwind and time integration methods, when the diffusive number $d$ is assumed to be small, $d \ll 1$.

method allows a larger time step than Adams-Bashforth as long as the first-order upwind scheme is used. For the second-order upwind scheme the Forward Euler method is less suitable, while the Adams-Basforth method allows a larger time step for this spatial scheme. The lower stability limit for the B3 upwind scheme seems to be a major disadvantage. However, the B3 scheme is needed in situations where using the B2 scheme results in an undesired amount of damping of the fluid motion due to artificial viscosity.

\section{Cell labeling method}

Before describing the discretisation of the equations, it is worthwhile to describe the cell labeling first. The variable $F_{b}$ describes the fraction of a grid cell open for fluid, while the variable $F_{S}$ describes the fraction of a grid cell filled with the liquid phase. The Navier-Stokes equations are solved in grid cells containing at least one of the fluids.

Every grid cell is given a label to distinguish between boundary, air and fluid. Two classes of labeling exist: geometry cell labels and fluid cell labels.

The geometry labeling at each time step divides the cells into three classes:

F(low) cells: All cells with $F_{b} \geq 0$

$\mathbf{B}$ (oundary) cells: All cells adjacent to a $\mathbf{F}$-cell

(e)X(ternal) cells: All remaining cells

The free-surface cell labeling is a subdivision of the $\mathbf{F}$ cells, consisting of 3 subclasses:

E(mpty) cells: All cells with $F_{s}=0$

S(urface) cells: All cells adjacent to an E-cell

F'(luid) cells: All remaining F-cells
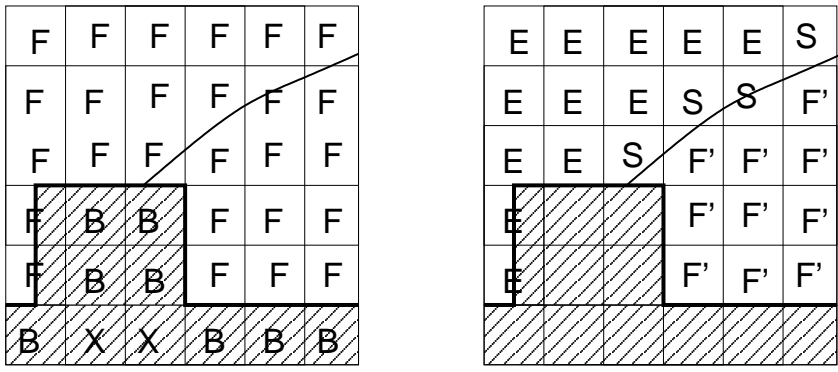

Figure 4. Geometry cell labeling (left) and free-surface cell labeling for wave impact on a rectangular container

Figure 4 shows an example of geometry cell labeling and free-surface cell labeling for wave impact from the right on a rectangular container.

The labeling system is retained in the two-phase model. In the one-phase model the empty cells were truly empty, as no computations were performed there, while boundary conditions for the free surface were prescribed around surface cells. In the two-phase model, however, the empty cells contain the fluid with the smallest density. The Navier-Stokes equations are also solved in these cells.

\section{Solving the pressure}

The pressure is calculated by means of a Poisson equation, which is formulated by taking the divergence of the momentum 
equation (5). The term $\nabla \cdot \mathbf{u}^{n+1}$ is then substituted into the mass conservation equation (4) to acquire the pressure Poisson equation:

$$
d t \nabla \cdot\left(\frac{1}{\rho^{n}} \nabla p^{n+1}\right)=\frac{1}{\rho^{n}} \frac{\rho^{n+1}-\rho^{n}}{d t}+\frac{\mathbf{u}^{n}}{\rho^{n}} \cdot \nabla \rho^{n}+\nabla \cdot \tilde{\mathbf{u}}^{n},
$$

with $\tilde{\mathbf{u}}$ the 'intermediate' velocity including the convective, diffusive and force effects. The right-hand side of the pressure Poisson equation (8) contains a $\rho^{n+1}$-term originating from the continuity equation. An equation of state will be necessary to replace the new density value $\rho^{n+1}$. The handling of this term in combination with the second (density gradient) term requires more attention and has been described earlier in [8]. The pressure Poisson equation finally reduces to

$$
d t \nabla \cdot\left(\frac{1}{\rho^{n}} \nabla p^{n+1}\right)=\frac{F_{b}^{n}-F_{s}^{n}}{F_{b}^{n}} \frac{1}{\rho^{n}}\left(\frac{\rho_{g}^{n+1}-\rho_{g}^{n}}{d t}+\mathbf{u}^{n} \nabla \rho_{g}^{n}\right)
$$

The remaining derivatives of the gas densities in eq. (9) do not contain large jumps anymore, as these derivatives are only determined by the compression and expansion of the gas phase. The gas density is substituted using the polytropic equation of state:

$$
\frac{\rho_{g}^{n+1}}{\rho_{g, 0}}=\left(\frac{p_{g}^{n+1}}{p_{g, 0}}\right)^{\frac{1}{\gamma}}
$$

with $p_{g, 0}$ and $\rho_{g, 0}$ the reference gas pressure and gas density values. The polytropic coefficient $\gamma$ has a value of 1.4 for pure air. The atmospheric pressure and ambient density values are used for $p_{g, 0}$ and $\rho_{g, 0}$. After substituting the equation of state (10) into the Poisson equation (9), the density is linearized by a Newton approximation and transferred to the left-hand side of the Poisson equation.

\section{DENSITY AND FREE SURFACE DESCRIPTION \\ Free surface description}

The free surface is in principle described by the equation $F_{s}(\mathbf{x}, t)=0$, with the motion described by

$$
\frac{D F_{s}}{D t}=\frac{\partial F_{s}}{\partial t}+\mathbf{u} \cdot \nabla F_{s}=0
$$

The displacement of the free surface is done in two steps, first the free surface is reconstructed and after that it is advected to the new position. The reconstruction of the free surface occurs by the Simple Linear Interface Calculation (SLIC) algorithm. This means that the interface only consists of line segments that are constructed parallel or perpendicular to the major flow axes. A characteristic drawback of the SLIC method is the unphysical creation of flotsam ('floating wreckage') and jetsam ('jettisoned goods'). These terms are appropriate for isolated, submesh-size droplets disconnected from the free surface because of errors induced by the free surface reconstruction. To prevent from this flotsam and jetsam, a local height function has been introduced earlier $[2,6]$. For the original VOF method without height function, the VOF-values are rounded off at the end of the displacement algorithm (values below zero are reset to zero and values above one are reset to one), leading to significant losses in liquid mass. To determine the local height function, first the orientation
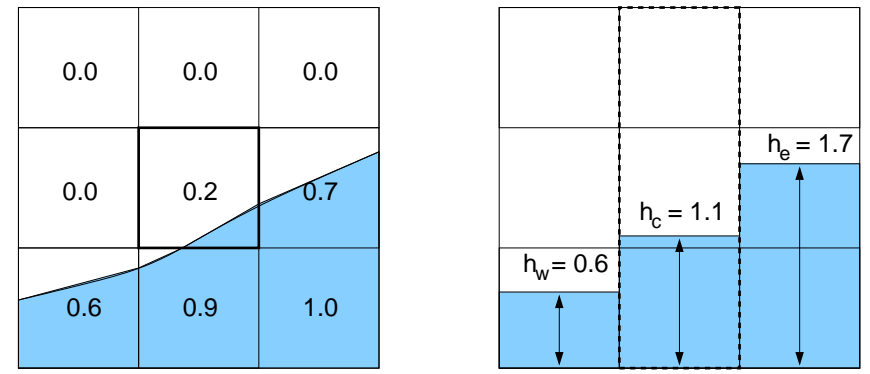

Figure 5. Construction of the local height function in a $3 \times 3$ block for a central S-cell.

of the free surface is determined (horizontal or vertical), depending on the values of $F_{S}$ in the surrounding block of cells. After that, the horizontal or vertical local height in each row or column in this block is determined by summing the VOF fractions (see figure 5). The local height function is applied in a $3 \times 3$ block (in $2 \mathrm{D}$ ) or a $3 \times 3 \times 3$ block (in $3 \mathrm{D}$ ) of cells surrounding a surface cell. By using the local height function, flotsam and jetsam are no longer present. Also, the loss of water is much smaller than with the original VOF method [4]. After reconstruction of the free surface, the new VOF-function $F_{s}^{n+1}$ is determined by calculating fluxes over every cell face using a donor-acceptor method [3].

\section{Density at the free surface}

Particular attention should be paid to the discretisation of the density, that can vary up to factor of 1000. Given the staggered arrangement of grid variables, see figure 2, pressure and density are both located in the cell centers. For compressible two-phase flow, the density in the cell center is calculated as

$$
\rho=\frac{F_{s}}{F_{b}} \rho_{l}+\frac{\left(F_{b}-F_{s}\right)}{F_{b}} \rho_{g}
$$


so in fact the density is a function of the liquid cell fraction $F_{S}$ and pressure $p$. The density is coupled to the pressure value in the cell centers, however the staggered variable arrangement induces the need to calculate the densities at the cell edges as well. This is the case for both the pressure gradient and the diffusive term. The calculation of the density at cell edges is a major point of attention, as serious errors (spurious velocities, see figure 6(a)) may be generated due to an inconsistent averaging method.

A main issue for the density averaging is the width of the averaging stencil. Considering a cell edge at $i+\frac{1}{2}$ (see figure 7 ), the question is whether it is necessary to take the complete grid cells $i$ and $i+1$ into account or if it is sufficient to consider only the right half of cell $i$ and the left half of cell $i+1$. The spurious

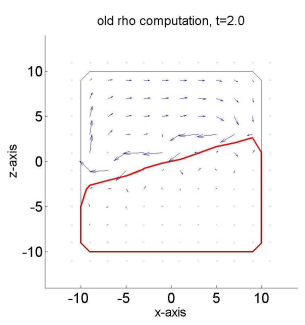

(a)

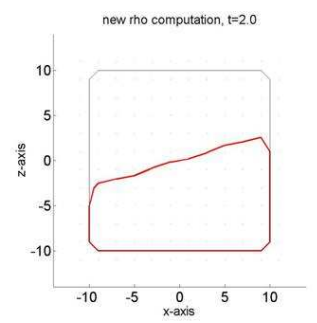

(b)
Figure 6. Spurious velocities due to simple averaging of density (left) disappear when a gravity-consistent density averaging is used (right).

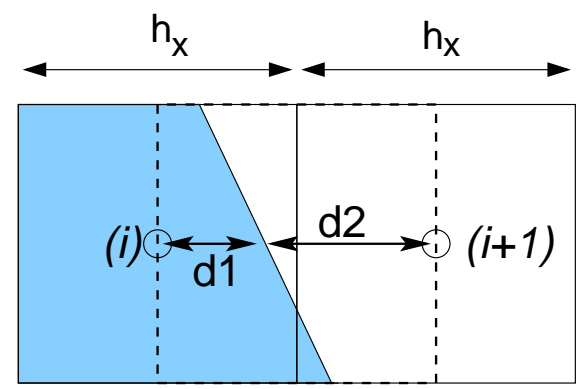

$(i+1 / 2)$

Figure 7. The gravity-consistent averaging method uses the normal distances $d_{1}$ and $d_{2}$ between the cell centers and the free surface.

velocities as visible in figure 6(a) were observed when the cellweighted averaging method (taking two complete grid cells into account to average the density) was used. To study the spurious velocities that were observed when applying cell-weighted averaging in COMFLOW, a hydrostatic fluid configuration (which should not lead to any flow) is defined. In the absence of any flow the momentum equation greatly reduces:

$$
\frac{1}{\rho} \frac{\partial(\rho \mathbf{u})}{\partial t}+\frac{1}{\rho} \nabla p+\frac{1}{\rho} \nabla \cdot(\rho \mathbf{u u})+\frac{1}{\rho} \nabla \cdot(\mu \nabla \mathbf{u})+\mathbf{F}=0
$$

so only the pressure gradient and the body force remain in the momentum equation. This means that the pressure gradient should be in balance with the gravitational force. According to eq. (13), the pressure gradient and body force should balance, i.e. $\nabla p=\rho \mathbf{F}$. Therefore, to ensure the balance between the pressure gradient and body force, the condition

$$
\nabla \times(\rho \mathbf{F})=0
$$

should be fulfilled, since $\nabla \times \nabla=0$. This provides a condition for the discrete average of the density. Condition (14) is fulfilled when the gravity-consistent averaging method is used, the density $\rho_{i+\frac{1}{2}}$ in figure 7 is then calculated by

$$
\rho_{i+\frac{1}{2}}=\frac{d_{1} \rho_{i}+d_{2} \rho_{i+1}}{d_{1}+d_{2}}
$$

Using this averaging method, the spurious velocities disappear, see figure 6(b).

\section{VALIDATION: SLOSHING IN LNG TANKS}

In this section the validation of the numerical model on sloshing in LNG tanks is described. As mentioned in the Introduction, the fluid behaviour in partially-filled LNG tanks needs investigation. To study this sloshing behaviour, both numerical simulations and model scale experiments have been carried out. For two test cases, with regular and irregular tank motion, the numerical and experimental results will be described.

\section{Setup of the model tests}

The model tests on scale 1:10 have been carried out to generate validation material for the numerical model. To match simulation and experiment, the numerical simulations are carried out on the same scale as the model experiments. The experimental setup has been designed as a 2D cross-section of a sloshing tank. Prior to the tests at DNV (Det Norske Veritas) in Oslo, the entire test setup (except the oscillator to move the tank) has been built and verified at MARIN.

The sloshing tank model was based on an LNG tank inside a No. 
96 LNG carrier. The inner side of the tank (the part open to fluid) has a width of $3897 \mathrm{~mm}$ and a height of $2697 \mathrm{~mm}$ on model scale. The tank is filled with water, while the front side and back side are made of perspex to enable visualisation of the fluid motion inside the tank.

Figure 8 shows screenshots of the sloshing tank for the $10 \%$
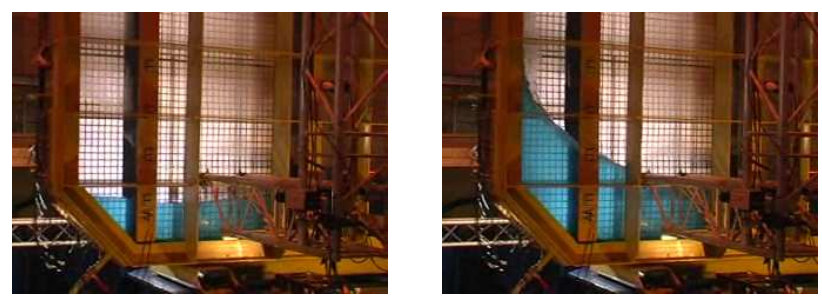

Figure 8. Screenshots of the 10 percent filling ratio sloshing model experiment. The left picture shows the fluid configuration at $t=0 \mathrm{~s}$, the right picture the configuration at $t=15 \mathrm{~s}$.

filling ratio case. As visible in these figures, the side walls of the tank are fitted with a number of measurement panels for pressure transducers, while the water height in the tank is measured by means of 12 water height probes. Furthermore, the tank is equipped with two cameras to monitor the fluid configuration.

The global motion time-traces of the tank, which are measured during the experiments, are used as input for the simulations. The motion of the tank is considered as a moving coordinate frame in the simulations to ensure similar conditions for simulations and experiment. The origin of the coordinate system is chosen at the center of the tank bottom.

\section{0 percent filling ratio, regular sway motion}

The oscillation period for this regular sway test case is $11.0 \mathrm{~s}$ on full scale and about $3.0 \mathrm{~s}$ in the experiment, resulting in about 400 impacts on each side wall during the 20-minute experiment. The fluid motion is relatively calm for this case, with a variation of 'bores' around the center of the tank and run-up motion near the side walls of the tank. Th experimental data have been compared with numerical simulations on a grid of $195 \times 1 \times 135$ cells, i.e. a grid space of $2.0 \mathrm{~cm}$. Figure 9 shows the water height development WH01 near the right tank wall. After 10 seconds of startup effects, the fluid motion is quite regular with slight variations in run-up at the tank wall. The predicted wave heights are close to the experimental values except for the two-phase simulation with the first-order upwind (B2) scheme. The artificial damping inherent to the first-order upwind scheme seems to hamper the water motion. This is particularly visible near the tank walls (see figure 9), where the run-up for the two-phase B2 simulation is

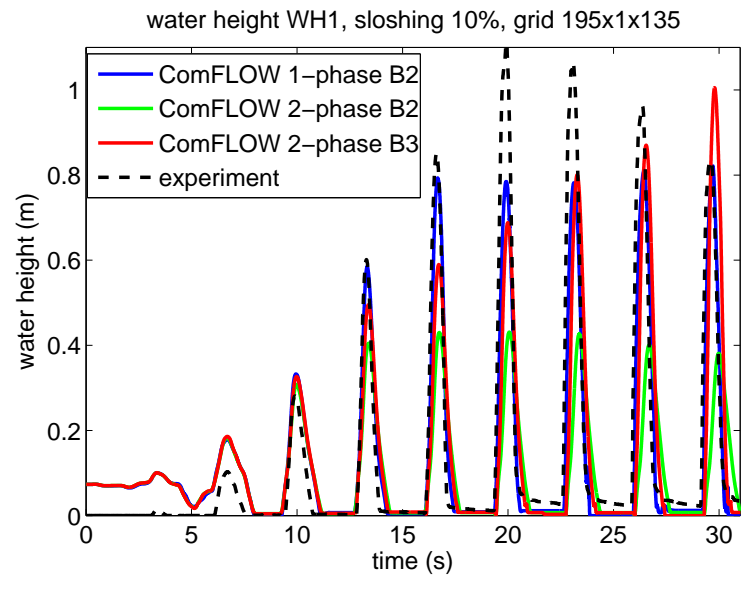

Figure 9. Water height development for 10 percent regular sway test at probe WH01, near the side tank wall.

much lower than in the experiment. The water height development at wave probe WH09, near the center of the tank, is shown in figure 10. Also for this wave probe, the artificial viscosity

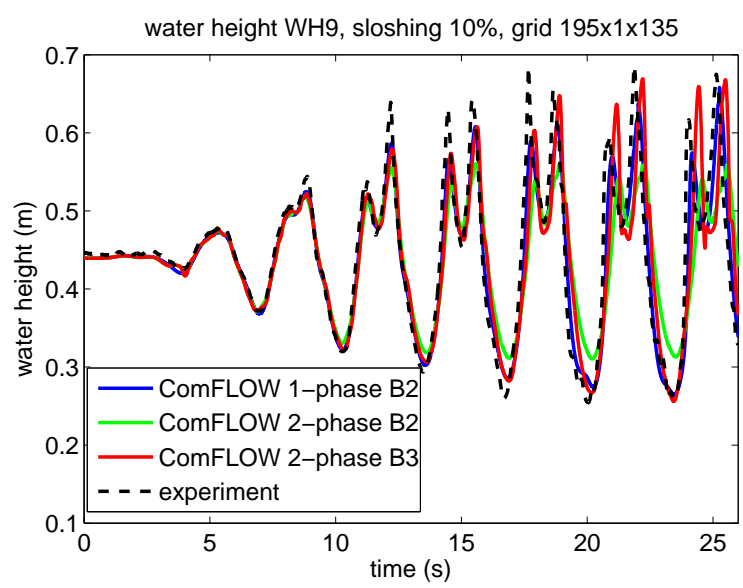

Figure 10. Water height development for 10 percent regular sway test at probe WH09, near the center of the tank.

in the air corresponding to the first-order upwind scheme leads to excessive damping of the water motion. So, to avoid these damping problems, it seems to be a better choice to apply the second-order upwind (B3) scheme. However, the B3 scheme is computationally more expensive. Since the artificial viscosity is proportional to the grid spacing, is it an alternative to keep using the B2 scheme but then on a finer grid? Figure 11 shows the results of a grid convergence study for the run-up at wave probe WH01. A rather fine grid of $390 \times 1 \times 270$ results in much more 


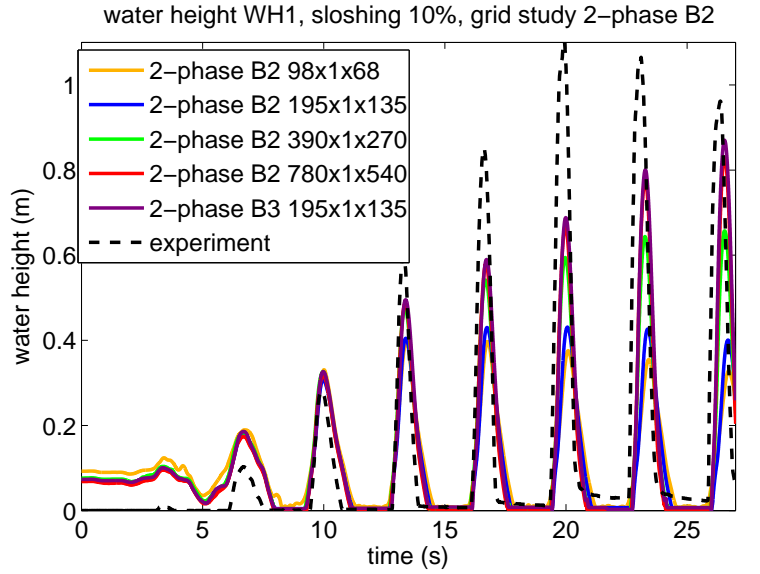

Figure 11. Grid convergence study for $10 \%$ filling ratio regular sway simulations at WHO1 $(x=1.8885 m)$.

run-up and is required to improve the water height prediction when the B2 scheme is applied. However, this grid refinement increases the computational costs by one order of magnitude due to the increased number of grid cells and the necessary reduction of the time step. Further refinement to a very fine grid of $780 \times 1 \times 540$ only slightly improves the prediction of the runup, and is hardly worth the increased computational effort.

An alternative for refining the grid to $390 \times 1 \times 270$ or even to $780 \times 1 \times 540$ cells is the use of the second-order upwind scheme. Comparing the water height for this B3 scheme with the results of the grid refinement for the B2 scheme in figure 11, the runup prediction by the $\mathrm{B} 3$ scheme on the coarse grid is still better. Hence, although applying the B3 scheme on the coarse grid leads to relatively high computational costs due to the lower CFL limit, it is still a 'cheaper' approach to predict the wave run-up for this test case than applying grid refinement for the B2 scheme.

The pressure development for transducer P01 in the lower tank corner in figure 12 shows a pattern that is related to the pattern visible for the water height development. After starting up, the pressures for the B2 two-phase simulation remain at a level that is clearly lower than the experimental values. For this case of regular sway motion, the differences between the B2 one-phase simulation and the B3 two-phase simulation are relatively small and both simulations agree quite well with the experimental pressure values. These small differences between the B2 one-phase and the B3 two-phase simulation can be addressed to the nature of this regular sway test case. The liquid motion is hardly affected by the dynamics of the compressible air surrounding the water, and no air entrapment has been detected during the experiments. Therefore, performing a one-phase simulation is sufficient for this low-filling ratio case with regular sway motion. For higher filling ratios and less repetitive motion time traces, however, the dynamics of the compressible second phase are much

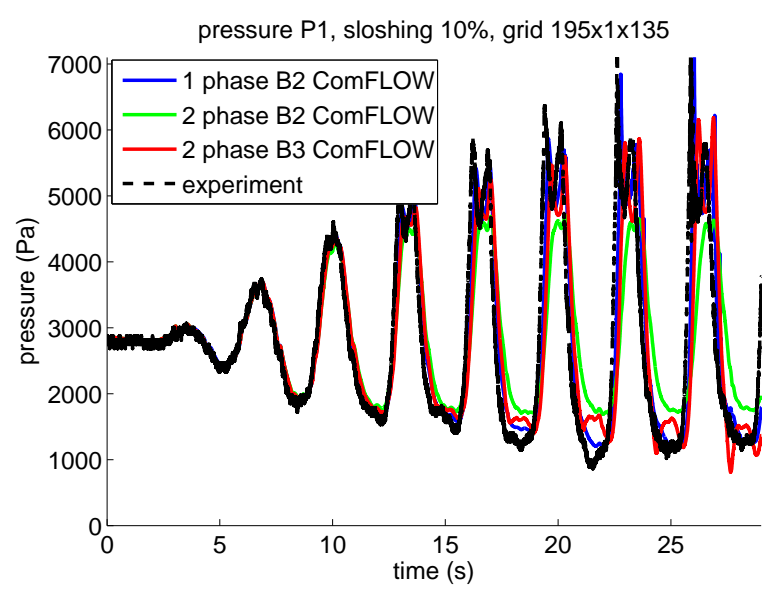

Figure 12. Pressure development for 10 percent regular sway test at transducer P01, in the lower tank corner.

more important.

\section{$\mathbf{1 0}$ percent filling ratio, irregular sway and roll motion}

For the irregular sway and roll motion case with a filling ratio of 10 percent, the oscillation period is $10.6 \mathrm{~s}$ on full scale and $3.2 s$ on model scale. The fluid motion is not continuously violent, although complex flow physics including air entrapment are sometimes visible during run-up at the side walls. The fluid motion is less repetitive than for the regular sway case, and wave breaking and air entrapment occur occasionally. During the first

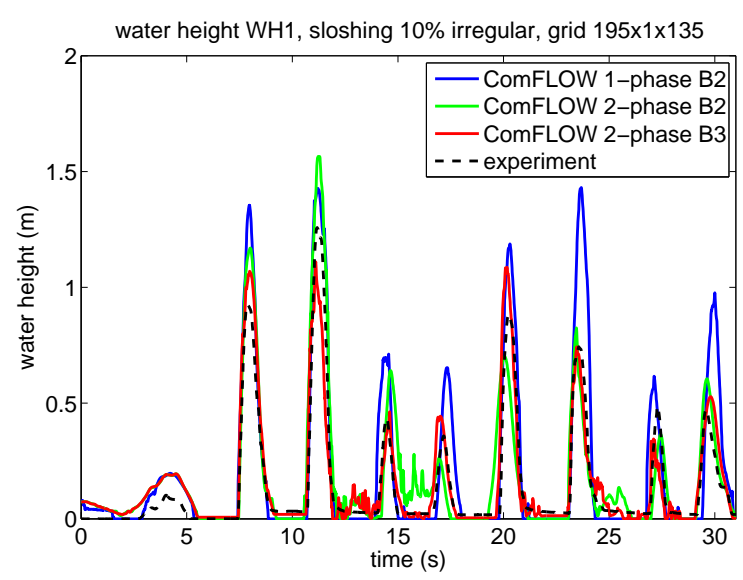

Figure 13. Water height development for 10 percent irregular motion test at probe WH01, near the side tank wall.

periods of this irregular motion case, the water height development is less predictable than for the regular sway case, see fig- 
ure 13. Simulation and experiment are in phase, but the onephase simulation overestimates the run-up against the tank wall. This discrepancy might be related to the water motion that is not damped at all by the second vacuum phase. The second phase is more viscous and heavier in the two-phase simulations, leading to a better agreement between the numerical and experimental water heights. Hence, for this irregular motion case, the wave run-up is predicted more accurately by the B2 and B3 two-phase simulations. The pressure development at the tank wall has also

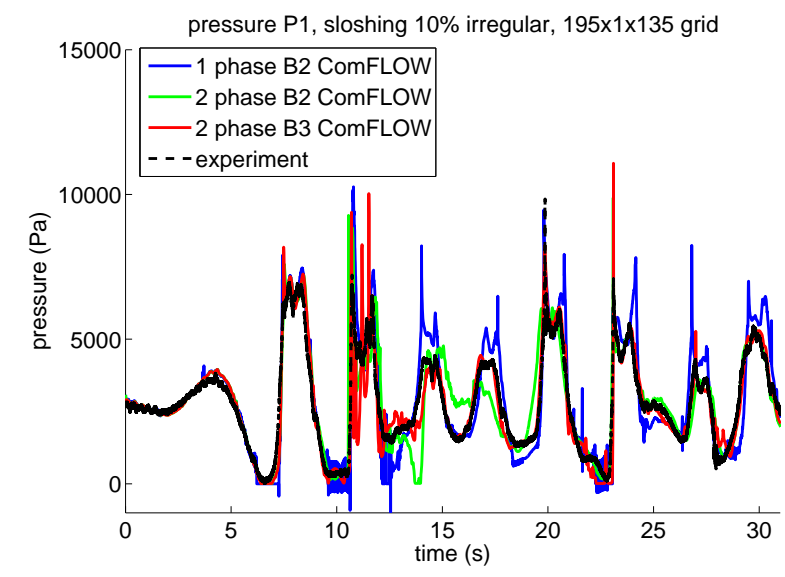

Figure 14. Pressure development for 10 percent irregular motion test at transducer P01, near the tank bottom.

been investigated for the irregular motion case. Figure 14 shows the pressure development in the lower tank corner. The pressure is overestimated (just as the water height) by the one-phase simulation. The two-phase simulations (B2 and B3) show a better agreement with the experimental data, although short-during pressure spikes occur as well in these two-phase simulations.

\section{CONCLUSIONS}

The results of the validation of the compressible two-phase flow model on sloshing model experiments have been presented in this paper together with the ingredients of the numerical method. By modeling compressible two-phase flow it is possible to describe the dynamics of the second phase accurately by allowing compression and expansion of the air.

The main flow variables velocity, pressure and density are calculated in all grid cells. The discretisation in space and time is treated in principle by the first-order upwind scheme and the Forward Euler method, respectively. Particular attention has been paid to the calculation of the density at the free surface, as the density calculation at cell edges also affects the pressure calculation. By doing this in the right way, spurious velocities are prevented.

For different test cases, it has been shown earlier [7] that the simulation results from the two-phase model have a better agreement with available measurement results than the results from the corresponding one-phase model. Now the numerical model has been tested on more elaborate sloshing experiments on 1:10 model scale. This validation has been done for both regular sway motion, as in [8], and irregular sway and roll motion. The artificial viscosity related to the first order upwind scheme appears to be an important factor, as it may lead to excessive damping of the fluid motion. The second order upwind scheme is less dissipative, but requires the use of the Adams-Bashforth time integration method. For calm weather conditions in combination with low tank filling ratios, the one-phase model gives a good prediction of water heights and pressure levels. However, for more irregular tank motion, related to more violent weather conditions, the results of the two-phase flow model are better, as the dynamics of the air phase plays a more important role in that case.

\section{ACKNOWLEDGMENT}

This research is supported by the Dutch Technology Foundation STW, applied science division of NWO and the technology programme of the Ministry of Economic Affairs in The Netherlands.

\section{REFERENCES}

[1] Greco, M., Colicchio, G., Faltinsen, O.M. Application of a 2D BEM-level set domain decomposition to the greenwater problem. 20th International Workshop on Water Waves and Floating Bodies, Longyearbyen, Norway, 2005.

[2] Gerrits, J., Veldman, A.E.P. Dynamics of liquid-filled spacecraft. Journal of Engineering Mathematics, 45:21-38, 2003.

[3] Hirt, C.W., Nichols, B.D. Volume Of Fluid (VOF) Method for the Dynamics of Free Boundaries. Journal of Computational Physics, 39:201-225, 1981.

[4] Kleefsman, K.M.T., Fekken, G., Veldman, A.E.P., Buchner, B., Iwanowski, B. A Volume-Of-Fluid Based Simulation Method For Wave Impact problems. Journal of Computational Physics, 206:363-393, 2005.

[5] Scardovelli, R., Zaleski, S. Direct Numerical Simulation of Free-Surface and Interfacial Flow. Annual Review of Fluid Mechanics, 31:567-603, 1999.

[6] Veldman, A.E.P., Gerrits, J., Luppes, R. Helder, J.A., Vreeburg, J.P.B. The numerical simulation of liquid sloshing on board spacecraft. Journal of Computational Physics, 224:82-99, 2007.

[7] Wemmenhove, R., Loots, G.E., Luppes, R., Veldman, A.E.P. Modeling two-phase flow with offshore applications. 
24th Offshore Mechanics and Arctic Engineering Conference, paper 67460, Halkidiki, Greece, 2005.

[8] Wemmenhove, R., Luppes, R., Veldman, A.E.P., Bunnik, T. Numerical Simulation of Sloshing in LNG Tanks with a Compressible Two-Phase Model. 26th Offshore Mechanics and Arctic Engineering Conference, paper 29294, San Diego, USA, 2007.

[9] Yabe, T., Xiao, F., Utsumi, T. The constrained interpolation profile method for multiphase analysis. Journal of Computational Physics, 169:556-593, 2001. 